\title{
Dioxygen Binding and Sensing Proteins
}

Darío A. Estrín, ${ }^{1}$ F. Javier Luque, ${ }^{2}$ Govindasamy Ilangovan, ${ }^{3}$ and Jay L. Zweier ${ }^{3}$

1 Departamento de Química Inorgánica, Analítica y Química Física/INQUIMAECONICET, Facultad de Ciencias Exactas y Naturales, Universidad de Buenos Aires, Buenos Aires, Argentina.

2 Department of Nutrition, Food Science and Gastronomy, Faculty of Pharmacy and Food Sciences, and Institute of Biomedicine, Campus Torribera, University of Barcelona, Santa Coloma de Gramenet, Spain.

3 Division of Cardiovascular Medicine, Department of Internal Medicine, Davis Heart and Lung Research Institute, The Ohio State University College of Medicine, Columbus, Ohio, USA. 


\begin{abstract}
Oxygen binding proteins (O2BIP) have been actively investigated for the past five decades due to their rich redox chemistry and function as 02 carriers in blood cells, as well as their function as gasotransmitters and sensors that modulate cellular signaling. A series of meetings on the periodic advances in the knowledge gained in the field of globin structure and function are conducted typically on a biannual basis. In the fall of 2018, the XXth International Conference was conducted, and very important articles with breakthrough discoveries were presented and very enthusiastically discussed. This was yet another highly successful meeting in the series. Select articles from this meeting were recently reviewed, updated, and published over several issues of Anti- oxidants and Redox Signaling, as Forum articles communicating the latest advances in this important area of redox biology. This Forum editorial introduces these articles and highlights their scientific significance in advancing the field. Each of these articles grew out of lectures presented in the meeting, and appears either as an original contribution or a comprehensive review in the journal. Overall, the articles published in the Forum provide in-depth details on the recent developments in the field as well as point the way to future directions. These Forum articles thus serve as an important summary of progress and the ongoing direction of this field, and serve to highlight recent advances in our understanding of 02BIP.
\end{abstract}

\title{
Keywords
}

oxygen, hydrogen sulfide, nitric oxide, globin 
The Antioxidant and Redox Signaling (ARS) Forum on Oxygen Binding and Sensing Proteins including Part A- Neuroblobin, and Parts B and C- Globins, contains part of the contributions presented at the XXth International Conference on Dioxygen Binding and Sensing Proteins (O2BIP), which was held in Barcelona, Spain, from September 3 to 6,2018 , organized jointly by the University of Barcelona and the University of Buenos Aires. This conference was held at the beautiful and historic Institut d'Estudis Catalans, which is located in the ancient building of The Hospital de Sant Pau, founded in 1401. This Conference was part of a series of Conferences on Oxygen Binding Proteins that began in 1966, and was followed by successful meetings held at Hamburg, Sheffield, Parma, Aarhus, and Naples, among other locations. About 130 leading scientists from all over the world attended the 2018 Conference in Barcelona.

The field of oxygen binding proteins started with the pioneering studies on myoglobin $(\mathrm{Mb})$ and hemoglobin $(\mathrm{Hb})$, which gave rise to numerous efforts in biochemical, biophysical, physiological, and evolutionary studies since the 1960s. The relevance of these two proteins in our understanding of structure-function relationships in proteins cannot be underestimated. Indeed, $\mathrm{Mb}$ has often been referred to as "the hydrogen atom of biology" (2). Later on, the complexity of the globin universe was unveiled by the discovery of novel members of this family, such as neuroglobin ( $\mathrm{Ngb}$ ) and cytoglobin (Cygb), as well as proto- globin and the so-called truncated $\mathrm{Hb}$ family. The functions of these newly discovered proteins, as well as the identification of noncanonical functions of $\mathrm{Mb}$ and $\mathrm{Hb}$, represent a matter of intense research. The complexity that underlies the apparent simplicity of the common globin fold has also been highlighted by their reactivity toward other gaso-transmitters, such as nitric oxide (NO) and more recently hydrogen sulfide (H2S), which have been proposed to be relevant players related to the physiological role of globin proteins, and accordingly are also the subject of significant research (Fig. 1).

To cover the discussion of recent advances in these areas, the scientific sessions of the O2BIP meeting were held on the following topics: globins in health and disease; structure, function, and dynamics; nonvertebrate, bacterial, and plants oxygen binding proteins; vertebrate oxygen binding proteins; evolution of oxygen binding proteins; and interactions with oxygen, nitrogen, and sulfur species.

Following the spirit of previous O2BIP editions, the meeting was focused on continuing the multidisciplinary effort made on disclosing the relationships between structure, dynamics, and function of globins and oxygen-related proteins, covering all levels of organization from molecular and cellular to whole organisms. In this context, the conference was a Forum for the presentation of recent advances and progress in the field, well suited for strengthening the scientific cross-fertilization between complementary research areas and for catalyzing the fruitful cooperation between teams with distinct expertise. The synergy of these different expertises, ranging from physical and biochemical sciences, molecular and structural biology, physiology, evolutionary studies, and computer simulation, has been a distinctive aspect of this series of conferences and is reflected in the articles that make up this Forum. The articles of the Forum were edited by the meeting organizers F. Javier Luque and Dario A. Estrin along with the journal Forum editor Jay L. Zweier supported by a team of expert reviewers chosen from the meeting participants (Fig. 2), as well as by the ARS peer review process.

To start off the Forum, two articles by Olson and Fernandez et al. were published in the Volume 32, Issue 4. Dr. Olson's review (ARS, 2020, vol. 32, 228-246) provides an extensive summary of lessons learned from 50 years of globin research. He describes how $\mathrm{O} 2$ uptake in red blood cells is limited by diffusion in unstirred water layers 
adjacent to the cell surface and across cell-free layers adjacent to vessel wall. Then discussion moves on to how structural changes alter the $\mathrm{Hb}$ and $\mathrm{Mb} \mathrm{O} 2$ uptake. Particularly, mechanisms of selective hydrogen bonding to $\mathrm{O} 2$, but not to $\mathrm{CO} 2$, by the distal histidine at the E7 helical position and prevention of oxidation of the ferrous heme are discussed. Also, the channel formation by upward rotation of His(E7) side chain in $\mathrm{Hb}$ and $\mathrm{Mb}$ and its relevance to the migration of $\mathrm{O} 2$ molecule to reach the heme site is discussed. Finally, the article discusses the assembly of $\mathrm{Mb}$ and $\mathrm{Hb}$ by formation of molten globule intermediates. The discussion on future direction regarding how best we can engineer these molecules for more efficient function is very exciting. In the same Forum, an original Forum research communication discusses how Ngb, a relative newcomer to the globin family (1), acts as redox sensor. In the seminal report (ARS, 2020, vol. 32, 217-227), Ngb was found to be a compensatory protein in the E2 regulatory protein-activated intracellular pathway, devoted to the in- crease of cancer cell tolerance to reactive oxygen species (ROS) generation.

This Forum also published articles related to " $S$ " bonding and its effect on the function of globins. Boubeta et al. (ARS, 2020, vol. 32, 247-257) discuss how the sulfide species influence/compete with $\mathrm{O} 2$ or NO binding to the ferrous ion in globins. This is a very important subject, as gasotransmitters such as $\mathrm{H} 2 \mathrm{~S}$ are being increasingly recognized for their important role in human physiology (7). Binding of sulfide either as neutral $\mathrm{H} 2 \mathrm{~S}$ or ionic HS- has remained controversial. Many studies prove that while H2S binds to the iron core in globins, HS- is excluded by the neighboring ligands. A critical issue in this subject is the experimental difficulty in obtaining spectroscopic evidence to prove this either way. Another article by Jenson et al. (ARS, 2020, vol. 32, 258-265) in the same Forum discusses a possible role for met- $\mathrm{Hb}$ as carrier of $\mathrm{H} 2 \mathrm{~S}$ in the blood. This is another dimension to the story of $\mathrm{H} 2 \mathrm{~S}$ binding, which is known to be nonoxidative binding to heme. As met-Hb does not bind $\mathrm{O} 2$, the $\mathrm{H} 2 \mathrm{~S}$ binding by met$\mathrm{Hb}$ must be $\mathrm{O} 2$ independent. The article nicely discusses the kinetics of metHb and $\mathrm{H} 2 \mathrm{~S}$ binding in the blood, $\mathrm{pH}$ dependence, and so on, and emphasizes that the future direction of this field must solve critical issues to understand the pathological levels of sulfide in blood and their dangerous targets in the vasculature. Considering these four articles, this Forum summarizes structure-function relation- ships of globins and how sulfide binding should be critically considered in addition to $\mathrm{O} 2 / \mathrm{NO} / \mathrm{CO} 2$ dynamics at the heme core of globins.

Articles published in the second part of the Forum in Volume 32, Issue 6, focus on the latest understanding of the different disease models related to globins. While presentations on structure-function relationships were discussed enthusiastically in the meeting (as presented in Issue 4), presentations pertaining to various disease models related to globin dysfunction were also received with equal enthusiasm in the meeting. We edited four selected articles representing this section of the meeting as part of this Forum. The first article in this section, by Hade et al. (ARS, 2020, vol. 32, 351-362), describes how truncated $\mathrm{Hb}$, namely $\mathrm{HbN}$ and $\mathrm{HbO}$, modulates the survival of mycobacterium bacteria in tuberculosis. This original Forum contribution reports the discovery that truncated $\mathrm{Hbs}$ have their own auto-kinase activity that supports the survival of the bacterium in hypoxia. An- other interesting Forum research communication by Rochon et al. (ARS, 2020, vol. 32, 363-377) reports the discovery of a novel role of nitrate to generate NO by nitrate reductase activity of globins in heart development in zebrafish. The structure-function relationship of a globin-coupled sensor in nitrogen fixation bacteria Azotobacter vinelandii was reported by Germani et al. in this Forum (ARS, 2020, vol. 32, 378-395), a horizon of new insight in the globin field. A Forum review in this Forum by Giordano et al. (ARS, 2020, vol. 32, 396-411) 
discusses recent advances in determining the cold adaptation and globins in Pseudoalteromonas haloplanktis TAC125. As temperature is critical in protein folding and denaturing, including heme proteins such as globins, perfect protein folding may be found in species that exist in extreme temperatures of Antarctica. Therefore, the mystery of protein evolution remains challenging, despite thermodynamic analyses and computational approaches to understand protein evolution. This review, based on the computational and thermodynamic analyses, details the challenges and future direction and describes how compu- tational approaches can reveal new information and integrate the globin field.

Finally, two articles on NO regulation in blood vessels by Cygb and the role of Cygb in renal protection are published in this Forum. These articles discuss the advancement in our understanding of NO scavenging in the vascular bed and ROS scavenging in the kidney. The role of globins in NO metabolism in blood vessels and vascular tone regulation remains controversial. There are two major globin pathways recently reported to have important roles in NO dioxygenation in vessels that impact NO signaling and vessel tone. First, Cygb in smooth muscle cells was found to be very efficient in scavenging excess NO in blood vessels. Deletion of Cygb was found to greatly decrease NO scavenging in smooth muscle cells and blood vessels $(4,5)$. Another pathway involving the myoendothelial junction and $\mathrm{Hba}$ has also been proposed as a major mechanism of NO scavenging $(3,6)$. The current Forum review article by Zweier and Ilangovan (ARS, 2020, this Forum) discusses in- depth detail about the role of Cygb NO scavenging, the status of the field, and future directions. The second article is an original contribution by Randi et al. (ARS, 2020, this Forum) that details how ROS generation leads to apoptosis and loss of podocytes from kidney and how Cygb can regulate this pathway. This discussion of ROS quenching in the kidney is an emerging field in our understanding of Cygb and its antioxidant function. Elegant studies, with use of transcriptomic analyses, are presented showing that knocking down the Cygb gene altered many genes in podocytes altering the redox balance.

Overall this was a very successful meeting with very scintillating discussions as mentioned earlier. We hope that the published Forum articles, both reviews and original contributions, serve the scientific community in communicating the current understanding in the field of O2BIP along with recent exciting developments as communicated at the meeting. We also hope that these Forum articles will serve to stimulate future research and advancement in the respective individual sub-fields within the globin superfamily.

\section{Funding Information}

We acknowledge the funding support from NIH/NHLBI grants R01HL131941 and R01HL135648 to J.L.Z. 


\section{References}

1. Fiocchetti M, Fernandez VS, Montalesi E, and Marino M. Neuroglobin: a novel player in the oxidative stress response of cancer cells. Oxid Med Cell Longev 2019: 6315034, 2019.

2. Frauenfelder H, McMahon BH, and Fenimore PW. Myoglobin: the hydrogen atom of biology and a paradigm of complexity. Proc Natl Acad Sci U S A 100: 8615-8617, 2003.

3. Lechauve C, Butcher JT, Freiwan A, Biwer LA, Keith JM, Good ME, Ackerman H, Tillman HS, Kiger L, Isakson BE, and Weiss MJ. Endothelial cell alpha-globin and its molecular chaperone alpha-hemoglobin-stabilizing protein regulate arteriolar contractility. J Clin Invest 128: 5073-5082, 2018.

4. Liu X, El-Mahdy MA, Boslett J, Varadharaj S, Hemann C, Abdelghany TM, Ismail RS, Little SC, Zhou D, Thuy LT, Kawada N, and Zweier JL. Cytoglobin regulates blood pressure and vascular tone through nitric oxide metabolism in the vascular wall. Nat Commun 8: 14807, 2017.

5. Liu XP, Follmer D, Zweier JR, Huang X, Hemann C, Liu KR, Druhan LJ, and Zweier JL. Characterization of the function of cytoglobin as an oxygen-dependent regulator of nitric oxide concentration. Biochemistry 51: 5072-5082, 2012.

6. Straub AC, Lohman AW, Billaud M, Johnstone SR, Dwyer ST, Lee MY, Bortz PS, Best AK, Columbus L, Gaston B, and Isakson BE. Endothelial cell expression of haemoglobin alpha regulates nitric oxide signalling. Nature 491: 473-477, 2012.

7. Sunzini F, De Stefano S, Chimenti MS, and Melino S. Hydrogen sulfide as potential regulatory gasotransmitter in arthritic diseases. Int J Mol Sci 21, 2020. 
FIG. 1. Schematic of the primary lo- cations, functions, and roles of various globins in normal or pathophysiology as discussed in the Forum articles of the XXth International Conference on O2BIP. All the molecules that are re- ported to bind $\mathrm{Fe} 2+/ 3+$ of the heme (such as $\mathrm{O} 2, \mathrm{NO}$, and $\mathrm{H} 2 \mathrm{~S}$ ) bind to the free li- gand position (for the fivecoordinate globins), or displace an internal ligand (for the six-coordinate globins). CM, cardiac muscle; Cygb, cytoglobin; GbX, globin-X; H2S, hydrogen sulfide; Hb, hemoglobin; Mb, myoglobin; MEJ, myoendothelial junction; Ngb, neuroglo- bin; NO, nitric oxide; O2BIP, dioxygen binding and sensing proteins; RBC, red blood cells; SM, skeletal muscle; SMC, smooth muscle cells.

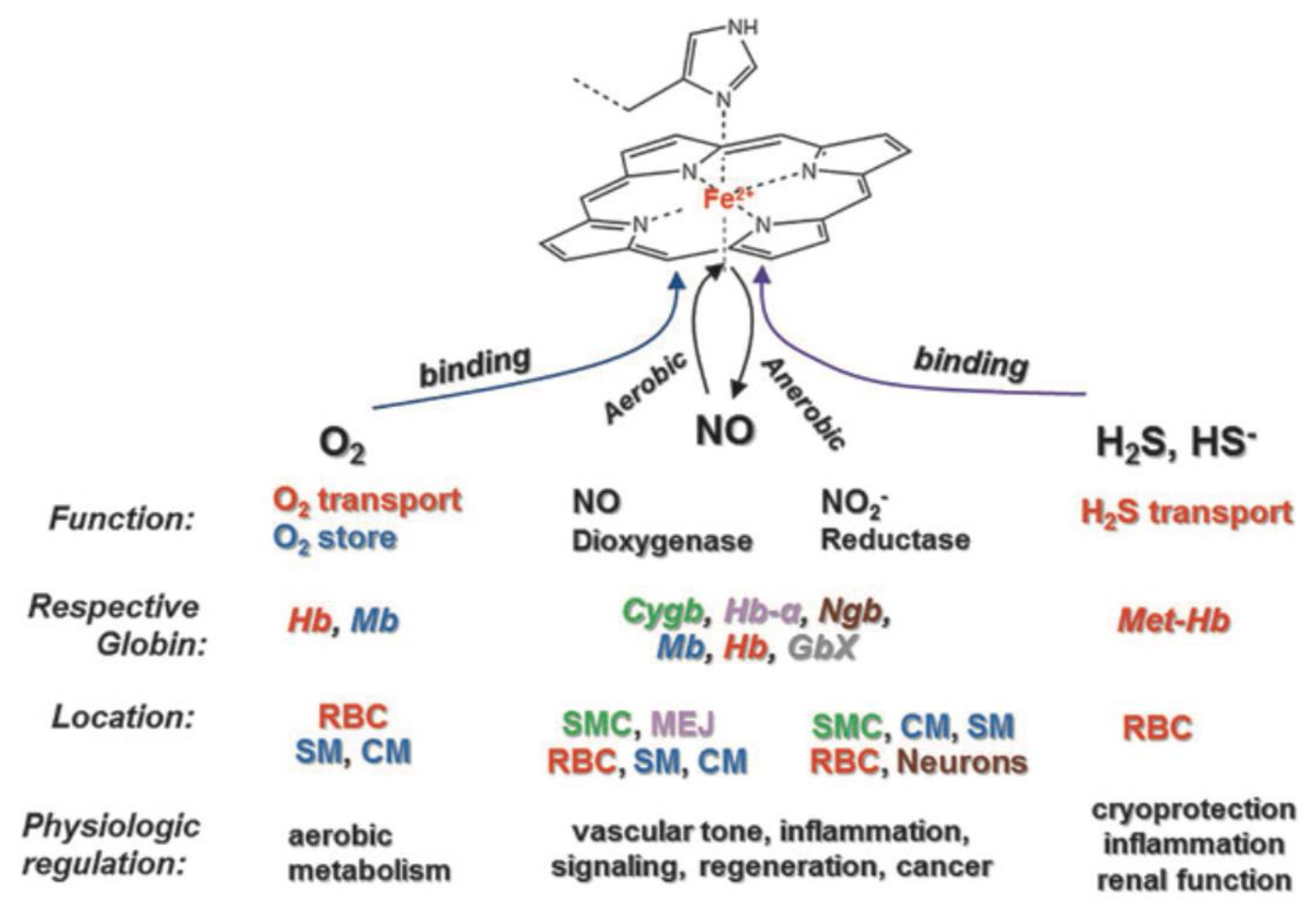


FIG. 2. A photograph of the participants in the XXth International Conference on Oxygen Binding and Sensing Proteins held at the historic Institut d'Estudis Catalans in Barcelona, Spain.

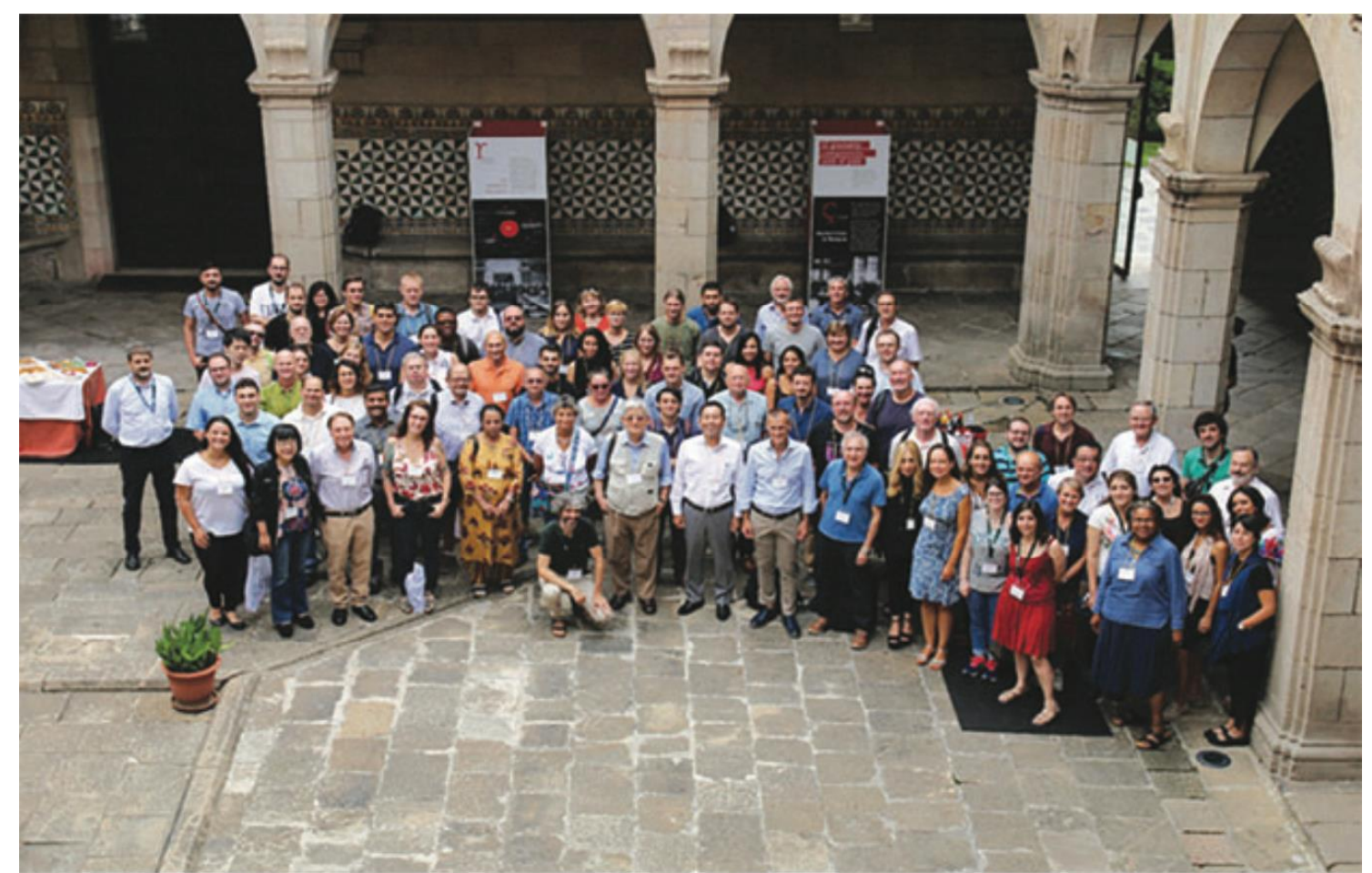

